\title{
Vivencias del paciente renal en la transición de diálisis peritoneal a hemodiálisis: estudio fenomenológico
}

\author{
Reyes Fernández Díaz ${ }^{1,2}$, Miguel Núñez Moral ${ }^{1,2}$, Beatriz Peláez Requejo ${ }^{1,2}$, Mónica Fernández Pérez ${ }^{1}$, Manuela \\ Rábano Colino ${ }^{1}$ \\ ${ }^{1}$ Enfermera/o de Diálisis Peritoneal. Unidad de Gestión Clínica de Nefrología. Hospital Universitario Central de \\ Asturias. Oviedo. España \\ ${ }^{2}$ Grupo de investigación en Cuidados en Nefrología. Instituto de Investigación Sanitaria del Principado de Asturias. \\ España
}

\section{Resumen}

Introducción: El cambio de modalidad dialítica evidencia un acontecimiento de gran estrés e incertidumbre cuando no es una transferencia deseada, y el cual se describe como un proceso de transición, donde la persona se mueve de un estado de la vida a otro, implicando el dominio de nuevos roles, conocimientos y habilidades para regresar al estado de equilibrio previo al cambio.

Objetivos: Describir la experiencia de los pacientes que transitan de diálisis peritoneal a hemodiálisis.

Material y Método: Estudio cualitativo, descriptivo, de orientación fenomenológica. Nueve informantes elegidos por muestreo de conveniencia fueron sometidos a entrevista semiestructurada en el domicilio. El desarrollo analítico resultó de un proceso deductivo-inductivo del que surgieron los ejes temáticos en los que se articuló la vivencia de los participantes. Se obtuvo la autorización del Comité Ético y los hallazgos fueron confirmados por los informantes.

Resultados: Emergieron 3 temas principales tales como, el significado de la experiencia de transición, las fases del proceso de la transición, y los cambios del ser en transición como fueron las modificaciones físicas, psíquicas, sociales y familiares.

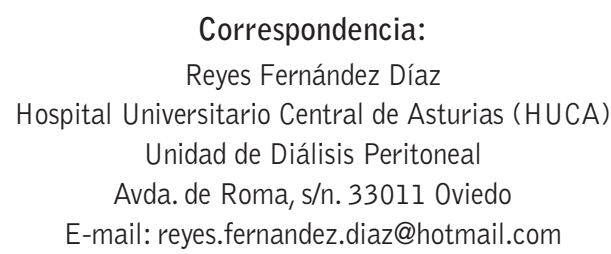

Conclusiones: La transición de diálisis peritoneal a hemodiálisis se revela como una vivencia vulnerable que denota cambios en el estado de salud y en las relaciones de roles y habilidades, asimismo, requiere cambio en los patrones de comportamiento de los participantes $y$, por tanto, resulta en la necesidad de redefinir un sentido de sí mismos.

PALABRAS CLAVE: acontecimientos que cambian la vida; cuidado de transición; diálisis renal; investigación cualitativa, atención de enfermería.

\section{Experiences of renal patient in the transition from peritoneal dialysis to hemodialysis: Phenomenological study}

\section{Abstract}

Introduction: The change of dialytic modality is an event of high stress and uncertainty when it is not desired, and which is described as a transition process, where the person moves from one state of life to another, involving the domain of new roles, knowledge and skills to return to the state of equilibrium prior to change.

Objectives: To describe the experience of patients who change from peritoneal dialysis to hemodialysis.

Material and Methods: Qualitative, descriptive study, based on the phenomenology. Nine informants selected by convenience sampling were subjected to semi-structured interview at home. The analytical development resulted from a deductive and inductive 
process from which emerged the thematic axes in which the experience of the participants was articulated. The authorization of the Ethics Committee was obtained and the findings were confirmed by the informants.

Results: Three main themes emerged: the meaning of the transition experience, the phases of the transition process, and the changes of the being human in transition in terms of how were the psychic, physical, social and family modifications.

Conclusions: The transition from peritoneal dialysis to hemodialysis is revealed as an experience of great vulnerability, which denotes changes in the health status, and in the relation between roles and skills, and it also requires changes in the behavior patterns of the participants and, therefore, results in the need to redefine a sense of themselves.

KEYWORDS: life change events; transitional care; renal dialysis; qualitative research; nursing care.

\section{Introducción}

La entrada en diálisis es un acontecimiento que genera cambios drásticos en la vida del paciente y su familia, precisando en muchos casos tener que seguir una terapia de diálisis de por vida para asegurar la supervivencia ${ }^{1,2}$. Diversas investigaciones cualitativas evidencian las importantes limitaciones físicas, psicológicas, y sociales, que amenazan la existencia de la persona durante la diálisis, como la pérdida de libertad, la depresión, la dependencia de otros, y las disrupciones familiares ${ }^{2-5}$. En base a esto, ciertos referentes legales, como la Ley 41/2002, 14 de noviembre de autonomía del paciente ${ }^{6}$ o el acuerdo de la European Dialysis and Transplant Association (EDTA) en la Conferencia de Munich de $1990^{7}$, amparan la libre elección del paciente acerca de la toma de decisión informada sobre el tratamiento renal sustitutivo que mejor se adapte a su estilo de vida, no obstante, a veces, los pacientes necesitan o deben ser transferidos de una modalidad dialítica a otra, contrariamente a su decisión ${ }^{8,9}$, pudiendo transitar de Hemodiálisis (HD) a Diálisis Peritoneal (DP) y viceversa, de diálisis a trasplante renal, o de fallo de injerto renal a diálisis, siendo posible transiciones múltiples entre los 3 estados $^{8,10}$. Desde la consideración, que las vivencias de cada persona son experiencias únicas, no es lo mismo confrontar al individuo ante el proceso de HD que $D^{3,4}$. Sauvé et al. comunican que la DP ofrece niveles más altos de autonomía, mejor preservación de la función renal residual, adecuación del rol laboral y mejor calidad de vida respecto a la $H D^{11}$, pero entrar en un programa de diálisis domiciliaria implica aprender a auto cuidarse, disposición que no todos los pacientes pueden o están capacitados para cumplir ${ }^{12}$. Partiendo del marco de referencia de Afaf Meleis, la transición es un proceso donde las personas son más vulnerables a riesgos que afectan su salud ya que implica pasar de un estado de la vida a otro con cambio en el comportamiento, adquirir cierto grado de autoconciencia y el dominio de nuevos conocimientos y habilidades para regresar al estado de equilibrio previo al cambio, y ello teniendo en cuenta los diversos condicionantes que pueden facilitar o limitar el progreso hacia una transición saludable como el nivel de salud, el status socioeconómico, las creencias o la disponibilidad de recursos $^{13}$. Existen evidencias de la desconexión de vínculos de los que dependen los sentimientos de seguridad de la persona durante el periodo de transición y como el proceso se vive con gran estrés, ansiedad e incertidumbre ${ }^{14,15}$. En este camino, se sugiere que la transición sea un proceso planificado, donde el paciente esté bien informado de las causas del cambio de modalidad con la suficiente antelación, en vistas a obtener una adecuada preparación física y psíquica ante la nueva terapia, y así pueda atenuarse el impacto en su calidad de vida ${ }^{16,17}$. A este respecto, la EDTA y la European Renal Association (ERA) publicaron en 2010, una serie de condiciones clínicas en las que debe planearse la transferencia entre modalidades de diálisis ${ }^{18}$. La revisión de la literatura constata la existencia de estudios que discuten la transición en el eje salud-enfermedad tal como, la vivencia de una enfermedad crónica ${ }^{19}$, el comienzo en diálisis ${ }^{20}$, la nueva vida con trasplante ${ }^{21}$, pero no se han encontrado investigaciones desde la perspectiva del paciente acerca de la experiencia de transición de una modalidad dialítica a otra. Por otro lado, se desconoce el significado transformador que supone la transición entre las terapias de sustitución renal y sus implicaciones en los diferentes componentes del ser humano, por lo que los resultados del presente estudio pueden fortalecer las bases para el desarrollo de un protocolo de atención en la transición, y así obtener resultados más satisfactorios en cuanto a calidad asistencial. Desde este escenario, el propósito del estudio fue describir la experiencia de transición de los pacientes que cambian de DP a HD.

\section{Material y Método}

Se realizó un estudio cualitativo descriptivo con abordaje fenomenológico, ya que se sustenta en la compresión profunda de la experiencia humana desde la perspectiva de la persona que la vivencia, lo que está 
en consonancia con la filosofía enfermera y la práctica del cuidado humanizado 22 y se enmarcó en la teoría de rango medio de las transiciones de Afaf Meleis ${ }^{13}$. La investigación se realizó en la Unidad de DP del Hospital Universitario Central de Asturias (HUCA) entre el periodo de septiembre de 2017 a mayo de 2018. EI universo de estudio fueron los pacientes diagnosticados de Enfermedad Renal Crónica Avanzada (ERCA) y que estaban en modalidad de DP en Asturias. Como criterios de inclusión se establecieron: pacientes autónomos para la técnica de DP, transitar de DP a HD, y permanencia mínima de dos meses en la antigua y nueva terapia renal. Los criterios de exclusión fueron: Pacientes en programa de ultrafiltración o con deterioro psíquico/ cognitivo. Se seleccionaron 20 participantes por muestreo de conveniencia, alcanzándose la saturación de datos en el informante nueve (Tabla 1). La captación se realizó cara a cara en la consulta de enfermería de DP mediante un proceso informado acerca del propósito de la investigación y de la confidencialidad de los datos. Ningún participante rechazó participar en el estudio.

Los datos se recogieron mediante entrevista semiestructurada, previamente se diseñó un guion de preguntas basado en la experiencia clínica del equipo investigador, que estableció una selección previa de temas como la vivencia en hemodiálisis, la vivencia en diálisis peritoneal, el cambio de terapia, los cambios de vida, la adaptación al cambio, las respuestas ante el cambio, y la red de apoyo. Posteriormente se estableció un proceso de suspensión del conocimiento antes del inicio de la investigación o bracketing, que consistió en realizar un pilotaje del guion de preguntas con dos informantes clave, y posponer la revisión bibliográfica hasta después de analizar los resultados del pilotaje. Se realizó una única entrevista a cada informante en su domicilio, sin la presencia de terceros, de una duración en torno a 45-60 minutos. La investigadora principal fue la persona encargada de realizar las entrevistas y analizar los datos, asimismo al ser enfermera asistencial en los cuidados nefrológicos de los participantes, pudo establecer una relación estrecha de cercanía con los mismos antes del inicio del estudio, lo que facilitó la verbalización de sentimientos y emociones en las entrevistas, aunque este estudio fue el primero de carácter cualitativo llevado a cabo por el equipo investigador. Desde el primer momento y previa autorización, las entrevistas se grabaron en formato audio-digital para su transcripción verbatim, igualmente se registraron notas en un cuaderno de campo sobre el metalenguaje, como el tono de voz, y la expresión facial y corporal. Los participantes confirmaron la compresión e interpretación de sus experiencias.

La estrategia analítica se fundamentó en un análisis de contenido mediante un paradigma de codificación deductivo-inductivo que se dividió en 5 etapas: 1) Elaboración de un marco conceptual con una lista inicial de códigos procedentes de la revisión bibliográfica acerca de las vivencias de los pacientes en diálisis; 2 ) Se realizaron varias lecturas de las entrevistas con objeto de segmentar el discurso de los informantes que contenía un significado relevante para el objetivo del estudio. 3) Se inició el proceso de codificación deductivo-inductivo que consistió en asignar a cada segmento, los códigos iniciales del marco conceptual, no obstante, se crearon nuevos códigos cuando emergieron ideas que complementaban o ampliaban los códigos iniciales. 4) Agrupación de los códigos en categorías para establecer diferentes ejes temáticos en los que se articuló la vivencia de los participantes en la transición de DP a HD. 5)

Tabla 1. Características sociodemográficas de los informantes.

\begin{tabular}{|c|c|c|c|c|c|c|}
\hline $\begin{array}{l}\text { INFORMANTES } \\
\text { (Pseudónimos) }\end{array}$ & SEXO & EDAD & $\begin{array}{l}\text { ESTADO } \\
\text { CIVIL }\end{array}$ & $\begin{array}{l}\text { SITUACIÓN } \\
\text { LABORAL }\end{array}$ & $\begin{array}{c}\text { *ESTANCIA } \\
\text { HD }\end{array}$ & $\begin{array}{l}\text { ** EPISODIOS } \\
\text { TRANSICIÓN }\end{array}$ \\
\hline Antonio & Masculino & 71 & Casado & Jubilado & 3 meses & 1 \\
\hline Jesús & Masculino & 76 & Viudo & Jubilado & 3 meses & 1 \\
\hline Blas & Masculino & 52 & Casado & Jubilado & 3 meses & 1 \\
\hline Cristina & Femenino & 61 & Casada & Labores domésticas & 2 meses & 2 \\
\hline Sonia & Femenino & 68 & Viuda & Jubilada & 2 meses & 1 \\
\hline Ángel & Masculino & 74 & Casado & Jubilado & 3 meses & 1 \\
\hline Olaya & Femenino & 46 & Casada & En activo & 2 meses & 1 \\
\hline Arturo & Masculino & 75 & Casado & Jubilado & 2 meses & 1 \\
\hline Oliver & Masculino & 54 & Casado & Jubilado & 4 meses & 1 \\
\hline
\end{tabular}

* Tiempo de permanencia en hemodiálisis.

** Número de transferencias de diálisis peritoneal a hemodiálisis. 
Elaboración de las matrices cualitativas que sintetizaron los resultados del estudio. Como apoyo en la fase analítica se dispuso del software MAXQDA.

Se obtuvo la aprobación del Comité de Ética de Investigación Clínica (CEIC) del Principado de Asturias con el $n^{\circ} 22 / 17$ y la autorización de la Dirección de Enfermería del HUCA. La intervención de los participantes fue libre y voluntaria, se preservó su identidad mediante la utilización de pseudónimos y, se obtuvo el consentimiento informado de los mismos.

\section{Resultados}

La experiencia de transición de DP a HD se articuló en tres categorías principales: El significado de la transición de DP a HD, las fases del proceso de transición de DP a HD, y los cambios del ser en transición.

\section{El significado de la transición de DP a HD.}

El significado de la transición de DP a HD (Tabla 2) se sustentó en interpretaciones fundamentadas en un cambio radical en el estilo de vida habitual.

"Es que fue un cambio radical de vida, que me separó de todo, y me afectó psicológicamente iiMucho!! iiMucho!! iiMucho!!..." (Entrevista Oliver: 148)

Los participantes concibieron la HD como una terapia más agresiva que la DP, que les generó un gran impacto emocional por la presencia de la sangre y por estar rodeados de más enfermos y, con la cual no se sintieron identificados.

"... al ver todos los enfermos allí me impresionaba más, el ver la sangre entrar y salir iMe impresionaba! es totalmente diferente, no es que me sentara mal, pero la hemodiálisis no es lo mismo que la peritoneal, es como más agresiva para el cuerpo ..." (Entrevista Sonia: 112)

Tabla 2. Matriz de ideas en el significado de la transición de diálisis peritoneal a hemodiálisis.

\begin{tabular}{|c|c|c|}
\hline CATEGORÍA & SUBCATEGORÍA & VERBATIM \\
\hline \multirow{6}{*}{$\begin{array}{l}\text { Significado de la } \\
\text { transición }\end{array}$} & Cambio de vida & $\begin{array}{l}\text { Cristina: "A mí me lo cambio iiiTOD0!!! la hemodiálisis" } \\
\text { Arturo: "... Ilevar dentro de lo que me sea posible, pues un poco de calidad de vida, que para } \\
\text { mí me la rompió totalmente ..." } \\
\text { Ángel: "Es que fue un cambio de vida, fue un cambio tan brusco" } \\
\text { Jesús: "La hemodiálisis fue un cambio total, en muchas cosas de mi vida" } \\
\text { Blas: "...sólo que tuve que cambiar iiiTOD0!!! debido a la hemodiálisis" } \\
\text { Antonio: "... mi vida dio un giro importante, cambiaron muchas cosas ..." }\end{array}$ \\
\hline & Agresiva & $\begin{array}{l}\text { Antonio: "... la hemodiálisis es ¡iMuy agresiva!! ..." } \\
\text { Sonia: "Es más dañina (la hemodiálisis), más agresiva" } \\
\text { Jesús: "Yo creo que es más dañina ¿no? te sacan la sangre" } \\
\text { Olaya: "Es más agresiva para el cuerpo" }\end{array}$ \\
\hline & Reforzamiento & $\begin{array}{l}\text { Arturo: "La hemodiálisis me valió para ponerme las pilas y hacerlo bien en peritoneal, y no } \\
\text { tener que volver (a hemodiálisis) ..." } \\
\text { Olaya: "Yo pienso que esta experiencia me reforzó, no pensé que era tan fuerte" } \\
\text { Oliver: "Me parece que fui bastante fuerte para afrontarlo, creía que no iba a poder" } \\
\text { Cristina: "No pensé que iba a ser tan fuerte, pero el saber que volvía a peritoneal me ayudó" }\end{array}$ \\
\hline & Traumático & $\begin{array}{l}\text { Sonia: "Para mí fue un trauma no poder hacer la peritoneal, que era a lo que yo estaba } \\
\text { acostumbrada" } \\
\text { Arturo: "... me produjo una especie de trauma ..." } \\
\text { Oliver: "Para mí fue bastante iiTraumático!! porque vino de repente" }\end{array}$ \\
\hline & Es diferente & $\begin{array}{l}\text { Cristina: "... es totalmente diferente, no es que me sentara mal, pero la hemodiálisis ¡iNo es } \\
\text { lo mismo que la peritoneal!!" } \\
\text { Jesús: "... entras en otra diálisis que es diferente, la hemodiálisis es distinta a la peritoneal, } \\
\text { ¡YO! no la quiero porque no es la mía, iiYo quiero la peritoneal!!" } \\
\text { Ángel: "... Es muy distinta (la hemodiálisis) a la peritoneal ..." }\end{array}$ \\
\hline & Sentirse preso & $\begin{array}{l}\text { Blas: "... ¡A ver! la hemodiálisis es como si estuvieras en libertad condicional y te mandaran } \\
\text { tres meses para la cárcel" } \\
\text { Cristina: "... la hemodiálisis te quita muchísima libertad ..." } \\
\text { Oliver: "Me sentí preso, de buenas a primeras no era capaz de salir de casa" } \\
\text { Arturo: "Te quita libertad y principalmente no tienes tiempo" }\end{array}$ \\
\hline
\end{tabular}


No obstante, contaron con el aliciente de que sabían que volvían a su terapia de origen y esa idea alentó su fortaleza personal ante la experiencia de la transición.

"... ¡Hombre! yo creo que al final ese tiempo en hemodiálisis me reforzó para hacer mejor las cosas en peritoneal, porque lo único bueno que tenía la hemodiálisis es que yo sabía que iba a volver a lo mío, a peritoneal" (Entrevista Blas: 215)

\section{Las fases del proceso de transición de DP a HD.}

El proceso de transición (Tabla 3; Figura 1) se manifestó en tres momentos bien definidos, en primer lugar, comprendió el desencanto con la nueva situación, y surgió la fase de desconexión respecto al rol actual, que supuso separarse de una vida basada en la libertad y el autocuidado aprendido en DP, aunque, por otro lado, supuso un rechazo profundo a estar en la nueva terapia.

"Tener que ir 3 veces a la semana a hemodiálisis te desconecta de tus rutinas, son muchas horas fuera de casa ... te sientes atrapado, sin libertad, dependes de la ambulancia ... a veces salía mareado porque me sacaban un poco más de líquido y sólo podía beber 1 litro ¡Claro! yo en casa lo tenía controlado, según lo que orinaba o sacaba por las bolsas (diálisis peritoneal), sabía lo que tenía que beber... la hemodiálisis no es para mí, no la quiero" (Entrevista Arturo: 73)

A medida que avanzó el proceso, los participantes no se adaptaron ni se integraron saludablemente a su nueva situación, sino que el nerviosismo debido a la inmovilidad que tenían que mantener en las sesiones y la falta de privacidad que percibían en la sala se revelaron como los principales componentes que reafirmaron la negativa a permanecer en HD.

"El estar rodeada con más enfermos, que yo bueno, estoy en mi casa, me organizo ;Yo! a mi manera, yo para mí, ¡No! ¡No la quiero!, (hemodiálisis)... aparte tampoco me podía mover porque lo llevaba en una pierna (catéter de hemodiálisis), entonces tenía que estar las cuatro horas o cinco horas sin poder moverme, para mí

Tabla 3. Matriz de ideas en las fases de transición de diálisis peritoneal a hemodiálisis.

\begin{tabular}{|c|c|c|}
\hline \multirow[t]{6}{*}{ CATEGORÍA } & SUBCATEGORÍA & VERBATIM \\
\hline & & $\begin{array}{l}\text { Cristina: "... notas que te separa de tu vida, no puedes seguir haciendo lo que hacías, yo sabía } \\
\text { que era para un par de meses, pero da igual ¡¡No quiero la hemodiálisis!!" }\end{array}$ \\
\hline & & $\begin{array}{l}\text { Olaya: "... no me dio tiempo a saber que el trasplante había fallado, cuando ya estaba en } \\
\text { hemodiálisis, a mí no me dio tiempo a reaccionar, yo no me enteré ..." }\end{array}$ \\
\hline & Desconexión & $\begin{array}{l}\text { Blas: "Yo antes iba a comer por ahí con los amigos, solía ir los viernes, y i¡Claro!!, con la } \\
\text { hemodiálisis ya no pude ..." }\end{array}$ \\
\hline & & Sonia: "No podía hacer las cosas que antes hacía, que yo me valía yo misma para todo" \\
\hline & & $\begin{array}{l}\text { Oliver: "Dejas de hacer lo de siempre, te falta tiempo, pierdes la vida que llevabas antes de la } \\
\text { hemodiálisis, para mí fue un trauma ya que tuve que dejar temporalmente de trabajar ..." }\end{array}$ \\
\hline \multirow{9}{*}{$\begin{array}{l}\text { Fases } \\
\text { de la transición }\end{array}$} & \multirow{5}{*}{ Inadaptación } & $\begin{array}{l}\text { Cristina: "Yo es que el enfrentarme a que tenía que estar tres horas y media acostada, inmó- } \\
\text { vil, no sé por qué me entraba tanto nerviosismo y nunca les decía nada a los profesionales allí, } \\
\text { pero ¡iYo estaba fatal!! me mentalizaba, pero no lo lograba" }\end{array}$ \\
\hline & & $\begin{array}{l}\text { Jesús: "Para esa diálisis (hemodiálisis) soy muy malo porque yo soy muy nervioso y estar tres } \\
\text { horas ahí en la máquina, estoy con una tensión que rompe modelos, estar ahí aguantándome } \\
\text { quieto, iiNo puedo!!" }\end{array}$ \\
\hline & & Antonio: "¡No! nunca me llegué a adaptar a la hemodiálisis" \\
\hline & & $\begin{array}{l}\text { Olaya: "... aparte tampoco me podía mover porque lo llevaba en una pierna (catéter de } \\
\text { hemodiálisis), entonces tenía que estar las cuatro horas o cinco horas sin poder moverme, para } \\
\text { mí fue bastante duro" }\end{array}$ \\
\hline & & $\begin{array}{l}\text { Ángel: "... nunca llegué a acostumbrarme, ibuf! qué nervioso me ponía cada vez que me } \\
\text { conectaban, iQué va, hombre!" }\end{array}$ \\
\hline & \multirow{4}{*}{$\begin{array}{l}\text { Nuevo rol } \\
\text { de vida }\end{array}$} & $\begin{array}{l}\text { Cristina: "... Allí (hemodiálisis) siento que estoy enferma, que yo sé que estoy enferma ¿no? } \\
\text { porque la enfermedad la tengo, pero no me siento tan enferma con la peritoneal" }\end{array}$ \\
\hline & & $\begin{array}{l}\text { Ángel: "... al tener que ir por la noche, tienes que dejar de hacer las cosas que hacías, bien } \\
\text { porque tienes que dormir o porque estás cansado ..." }\end{array}$ \\
\hline & & Arturo: "... noté pérdida de libertad y que estás más atado ..." \\
\hline & & $\begin{array}{l}\text { Antonio: "... mi vida era de moverme mucho de aquí para allá y entonces pues ¡Nada! tuve } \\
\text { que dejarlo" }\end{array}$ \\
\hline
\end{tabular}




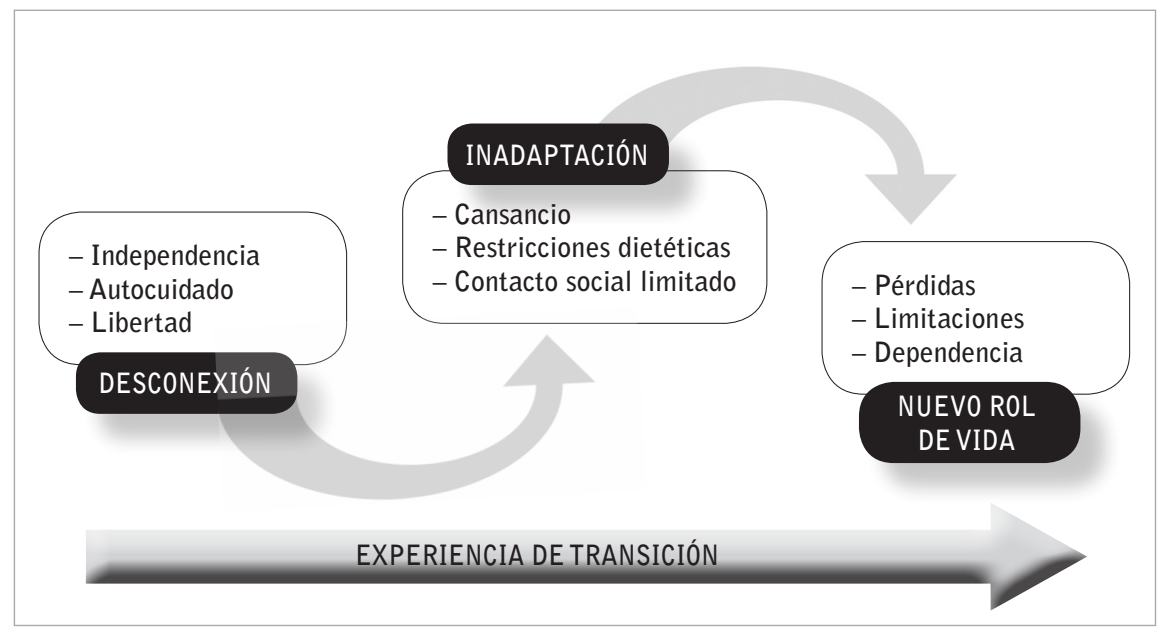

Figura 2. Proceso de transición de diálisis peritoneal a hemodiálisis.

fue bastante duro (silencio), yo estaba deseando volver a lo mío (diálisis peritoneal)" (Entrevista Olaya: 34).

Finalmente, emergió un nuevo rol de vida que asociaron a profundas pérdidas, limitaciones y dependencia respecto a terceras personas, transcendiendo la identidad de sentirse presos en vida.

"Te acostumbras y vas asumiendo que esto es lo que hay, pasas a estar pendiente de ir y venir del hospital... pierdes mucho tiempo para hacer otras cosas, $y$ tienes que beber menos líquidos y cuidar más la dieta, pero bueno fue temporal, no para siempre ... yo sabía que regresaba a peritoneal, pero notas mucha falta de libertad, al menos yo eso lo noté muchísimo, te sientes como preso" (Entrevista Antonio:104)

\section{Los cambios del ser en transición.}

Los participantes describieron como se percibieron a sí mismos y los cambios que habían experimentado a través de la transición (Tabla 4). La falta de energía o el cansancio emergió como el cambio físico más evidente que les impedía realizar actividades físicas o sociales, hasta el punto de tener que renunciar a ellas.

"Dejé de salir a caminar porque cansaba, iiMucho!! iiMucho!!, de hecho, mi vida era de moverme mucho de aquí para allá y entonces pues, iNada!, tuve que dejarlo (eleva los hombros como gesto de conformidad) ..." (Entrevista Antonio: 170)

Los cambios físicos en el cuerpo determinaron alteraciones en la imagen corporal y surgieron respuestas de miedo, dolor y respeto ante el catéter venoso central, en contraposición a la normalidad asignada al catéter peritoneal.

"...lo del cuello (catéter de hemodiálisis) me daba más respeto, ¡No sé!, tenía miedo que me saliera y ponerme a sangrar...en cambio con el de peritoneal ¡No! como está en la tripa..." (Entrevista Sonia: 112)

Se manifestó una identidad de enfermo, ya que se sintieron más enfermos en HD porque tomaron más conciencia de su enfermedad renal.

"Me costó entrar en hemodiálisis, porqué me sentí un enfermo de verdad, cuando yo en peritoneal no me noto enfermo, allí (hemodiálisis) ves a gente que está muy malina y piensas que a ti te va a pasar lo mismo, es como que tomas más conciencia de todo ..." (Entrevista Ángel: 75)

Se produjeron modificaciones en las necesidades básicas como la dieta, el aseo, la actividad física, o el sueño. Tener que seguir un régimen de alimentación acorde con los requisitos de la HD limitó el ritmo de vida habitual porque supuso reducir vida social.

"... porque esto (la hemodiálisis), ¡Claro!, (chasquido con la lengua) con la restricción de líquidos sales menos, y luego los demás, pues toman un vino, una cañita, y tú estás, que ni un vaso de agua, a un café corto, porque el líquido tiene que ser poco, entonces, dejas de salir por eso..." (Entrevista Olaya: 92)

Aprender a ducharse con un catéter que, ni se puede mojar ni se puede curar, despertó la intranquilidad e impotencia de los participantes que estaban acostumbrados a ejercer su autocuidado, alimentando la sensación de pérdida de control sobre el cuerpo y emergiendo la dependencia respecto a los profesionales.

"... pero con el otro (catéter de hemodiálisis) no es 10 mismo, porque no me dejaban curarlo, iiJo!! entonces tenía que tener cuidado de no mojarlo y eso era un roIlo, ¡Hombre! te ponen un plástico para que no entre el agua, pero ¡Da igual!, ¡No es lo mismo! ... ya no tienes la misma tranquilidad" (Entrevista Antonio: 54)

La restricción de actividades se consideró el factor más limitante en el día a día, y el cual tuvo una fuerte relación con la idea de cansancio que expresaron. 
Tabla 4. Matriz de ideas en los cambios del ser en transición.

\begin{tabular}{|c|c|c|}
\hline CATEGORÍA & SUBCATEGORÍA & VERBATIM \\
\hline \multirow{6}{*}{$\begin{array}{l}\text { Cambios } \\
\text { ser } \\
\text { en transición }\end{array}$} & Cambios físicos & $\begin{array}{l}\text { Oliver: "... en ese proceso, te sientes decaído, débil ..." } \\
\text { Ángel: "... salía bastante cansado de las sesiones." } \\
\text { Cristina: "... me levantaba mucho dolor de cabeza siempre ..." } \\
\text { Jesús: "... cansaba y no me apetecía hacer nada, estaba apático" } \\
\text { Sonia: "Salía agotada y el día después seguía cansada" }\end{array}$ \\
\hline & $\begin{array}{c}\text { Cambios } \\
\text { autoimagen }\end{array}$ & $\begin{array}{l}\text { Olaya: "... me sentía hinchada, iClaro!, llega el fin de semana, la pones (sesión hemodiálisis) } \\
\text { de viernes y hasta el lunes no vuelves a dializarte, yo el domingo estaba que no entraba en la } \\
\text { ropa ..." } \\
\text { Oliver: "Yo quería deshacerme del catéter en el cuello, era una cosa que me tenía enfermo" } \\
\text { Blas: "... para mí el poner esto (catéter de hemodiálisis) y encima en la vena, era lo más } \\
\text { gordo del mundo" }\end{array}$ \\
\hline & $\begin{array}{c}\text { Cambios } \\
\text { necesidades } \\
\text { básicas }\end{array}$ & $\begin{array}{l}\text { Antonio: "iiAllí!! (hemodiálisis) bebía menos porque me lo mandaban (carraspea), no porque } \\
\text { yo quisiera, ieh!" } \\
\text { Arturo: "Con aquella (hemodiálisis) apenas salía, cansaba" } \\
\text { Ángel: "Te ponen un plástico para que no se moje (catéter de hemodiálisis) pero tenía que } \\
\text { ayudarme mi esposa, porque yo sólo no me arreglaba muy bien, eso antes no me pasaba, con } \\
\text { éste (catéter de peritoneal), si se moja, pues se moja, luego lo curas y ya está" } \\
\text { Olaya: "...tenía el sueño cambiado y el día que descansaba (día de no hemodiálisis) me costa- } \\
\text { ba también dormirme por la noche" }\end{array}$ \\
\hline & $\begin{array}{l}\text { Cambios } \\
\text { psíquicos }\end{array}$ & $\begin{array}{l}\text { Cristina: "¡No sé! al ver todos los enfermos allí, me impresionaba más, el ver la sangre entrar } \\
\text { y salir ¡Me impresionaba!" } \\
\text { Jesús: "... estaba nervioso, porque me tenía que echar en aquella cama y estar quieto." } \\
\text { Antonio: "... al ir en ambulancia tardaba ¡¡Muchísimo más!!, me recogían a las doce de la } \\
\text { mañana y no regresaba a casa, hasta casi las ocho de la tarde, ijJo!!, eso era un agotamiento } \\
\text { de cabeza tremendo" } \\
\text { Ángel: "... me volví más irritable, todo me molestaba" }\end{array}$ \\
\hline & $\begin{array}{l}\text { Cambios } \\
\text { sociales }\end{array}$ & $\begin{array}{l}\text { Olaya: "...dejas de salir porque como no puedes beber mucho líquido, entonces no te presta } \\
\text { estar por ahí con alguien" } \\
\text { Oliver: "... me sentí preso también, de buenas a primeras, no puedes salir de casa." } \\
\text { Sonia: "Con la hemodiálisis no viajaba, aunque yo pudiera hacerla en otro sitio, en el destino } \\
\text { al que vaya, i¡No, No!!, no marchaba, tenía miedo que me pasara algo" } \\
\text { Ángel: "Yo temía a que los demás me vieron como un enfermo, pero es que yo no me siento } \\
\text { enfermo, bueno en hemodiálisis estuve más flojo y decaído, pero al volver a peri, volví a ser el } \\
\text { que era" }\end{array}$ \\
\hline & Cambios familiares & $\begin{array}{l}\text { Cristina: "Mi familia, mi marido y mis hijas fueron mi principal apoyo" } \\
\text { Sonia: "... el día antes, mi mujer ya tenía que estar poniendo el remojo, pa lo que ibas a } \\
\text { comer el día siguiente" } \\
\text { Arturo: "... ella (esposa) me llevaba en coche (a hemodiálisis) para que yo no tuviera que } \\
\text { estar pendiente de la ambulancia ..." } \\
\text { Oliver: "Procuraba no decirle nada a mi esposa para no preocuparla" }\end{array}$ \\
\hline
\end{tabular}

"... apenas salía de casa porque me notaba un poco cansado..." (Entrevista Antonio: 130)

Asimismo, la calidad del sueño empeoró en HD en relación a cuando tenían asignado el turno de la noche para dializarse.

"Ibas de noche, con lo cual ya no dormías, ni dormías allí ni dormías en casa cuando llegabas" (Entrevista Arturo: 34)
El nerviosismo se manifestó como el principal cambio que repercutió en la psique de los participantes, y el cual se avivó por la inmovilidad física que había que mantener durante la conexión a la máquina.

"Yo es que el enfrentarme a que tenía que estar tres horas y media acostada, inmóvil, no sé por qué me entraba tanto nerviosismo y nunca les decía nada a los profesionales, pero yo estaba fatal ...intentaba mentalizarme, pero no lo lograba" (Entrevista Cristina: 97) 
No obstante, destacó el cambio de carácter por no estar en la diálisis deseada y elegida, refirmándose el sentimiento de no sentirse identificados con la nueva terapia, tal y como reveló la siguiente evidencia.

"La diálisis te cambia el carácter, i¡Muchísimo!!, aquella la de la sangre (hemodiálisis), a mí me cambió más todavía, porque no era la mía" (Entrevista Blas: 296)

La vida social tornó un significado diferente durante la transición, la pérdida de libertad que manifestaron se trasladó a otro tipo de pérdidas y limitaciones que cohibieron la vida diaria de los participantes.

"Dejé de hacer cosas, lo primero dejé de ir a caminar por las mañanas porque me notaba muy flojo, no podía con el alma, y luego que yo iba en turno de tarde, y la mañana no me daba tiempo, entre que me levantaba tarde porque estaba cansando y que me venía a buscar la ambulancia, ya no tenía tiempo de nada, y al día siguiente descansaba isí!, pero no me apetecía" (Entrevista Oliver: 200)

Las actividades sociales se estancaron y surgió un contacto social limitado, bien por el cansancio o apatía que provenían de las sesiones, o bien por las restricciones dietéticas que generaron falta de energía en el cuerpo.

\footnotetext{
"Nosotros (esposa y paciente) a veces solemos salir a cenar, pero cuando estuve en hemodiálisis dejamos de hacerlo ... entre que me notaba algo cansando y tampoco podía comer ni beber como en peritoneal, pues nada, no solíamos iPa qué! ya no era lo mismo" (Entrevista Ángel: 82)
}

Se exteriorizó una identidad de enfermo social cuando se dieron cuenta de que su entorno podía identificarles como un ser enfermo, lo que coincidió con el rol que sentían que debían asumir en HD.

"... no se siente uno a un nivel así de los demás, de ser una persona normal, de pronto caes en otro status, iNo puedes!, estás limitado de todo, y estás como apenado, uno se apena porque tienes miedo a que los demás vayan a sentir pena de uno, i Vaya!!, una cosa que psicológicamente golpea muy fuerte ..." (Entrevista Oliver: 30 )

No obstante, encontraron un buen apoyo entre los profesionales que les atendían en HD, y con otros pacientes que se dializaban en la sala.
"Haces amistades, porque son muchas horas las que estás allí, además te sientes como un poco más arropado, al principio piensas que sólo eres tú y hasta que no entras allí (sala de hemodiálisis), no te das cuenta de lo que hay (silencio), y vas conociendo a más gente que tiene tú mismo problema" (Entrevista Ángel: 115)

Muchos factores que impactaron en su mundo social también afectaron al núcleo familiar de los participantes. La familia se reveló como la principal red de apoyo en el abordaje de la transición, principalmente la pareja o el cónyuge, ya que facilitaron el desempeño de actividades.

" No podía hacer ni la cama! tampoco era capaz de cocinar, tenían que hacerlo o la mi hija o mi nieta" (Entrevista Sonia: 11)

La red familiar experimentó cambios en sus roles, desde la forma de preparar los alimentos, pasando por la restricción de actividades, hasta la preocupación que emergió por no ver a los participantes con la misma imagen.

"Mi familia preocupada (suspira) porque me veían que no estaba igual que como estoy con la peritoneal" (Entrevista Antonio: 85)

"...yo suelo comer lechuga 3 días a la semana y tuve que volver a ponerla a remojo, tenía que estar pensando que iba a comer al día siguiente, si patatas o algo de verdura, para dejarlo antes en remojo, eso antes no me hacía falta" (Entrevista Sonia: 95)

Asimismo, la familia estableció fuertes lazos de protección con los participantes forjando cierta dependencia que no estaba presente en el rol de vida anterior.

"Podía ir en ambulancia y, i Jamás!!, i Jamás!!, me dejaron, como que estaba más protegida llevándome ellos (marido e hijas), que me sintiera yo mejor" (Entrevista Cristina: 113)

Mientras, los participantes sintieron la responsabilidad de permanecer fuertes y apoyar a la familia, intentando ser autosuficientes o ejerciendo cierto silencio emocional.

"... dentro de la familia nunca expresé como me encontraba por no afectar a los míos, porque yo no quería que mi familia sufriera lo que yo estaba pasando 
(chasquido con la lengua), lo que pudiese evitar lo evitaba" (Entrevista Ángel: 119)

\section{Discusión}

Vivir una experiencia de transición de DP a HD se reveló como una vivencia de gran vulnerabilidad que implicó cambios profundos de vida en la esfera holística de los participantes, para ellos, el cambio de terapia significó un cambio drástico de vida y reajustes de rutinas para adaptarse a la nueva realidad. Este es un hallazgo común en investigaciones que analizan las vivencias o experiencias de los pacientes cuando transitan de la enfermedad renal crónica a diálisis (Hutchinson ${ }^{23}$; Jonasson et al. ${ }^{5}$; Kaezer et al. ${ }^{24}$ ) pero que no se ha podido contrastar con estudios de transición entre modalidades de diálisis porque no se han encontrado en la revisión bibliográfica.

El proceso de transición se sintetizó en tres etapas: La desconexión, la inadaptación y un nuevo rol de vida. Schumacher y Meleis ${ }^{25}$ determinaron que, la característica más generalizada de la transición es la desconexión asociada con la interrupción de vínculos o puntos de referencia de los que depende los sentimientos de seguridad de la persona, Este aspecto, se confirmó en nuestras entrevistas, ya que los participantes identificaron la pérdida de libertad y la pérdida de control sobre los cuidados de su cuerpo como elementos claves que limitaban sus actividades cotidianas (Jonasson et al. ${ }^{5}$, Lindsay et al. $^{26}$ ). Estas limitaciones también surgen en el estudio de Moran et al. ${ }^{27}$, como la vida que viene con la HD detiene a los pacientes de las actividades que antes se daban por sentadas, aunque hay que destacar que se refiere al paso de pacientes de una situación de enfermedad a diálisis.

La transición no se experimentó uniformemente entre los participantes, pero hubo puntos en común, y uno de ellos es que, no fueron capaces de asociar una sensación de seguridad a la transición y, por tanto, no lograron una plena adaptación al proceso, bien por el nerviosismo que desencadenaba la terapia o por la situación de inmovilidad durante las sesiones. Este resultado de falta de integración contradice investigaciones orientadas a la teoría de las transiciones de Meleis et al. ${ }^{25}$, donde la finalización de una transición implica que la persona encuentra un periodo de mayor estabilidad a través del crecimiento personal, sin embargo, se localizó una referencia en el estudio de Arman y Rehnsfedlt ${ }^{28}$, donde las mujeres se clasifican como "estancadas" en el pro- ceso de transición, porque no son capaces de adaptarse e integrar el cáncer en sus vidas, y por tanto, cuestiona cómo es el papel que desempeña el profesional en el abordaje de la transición, y si éste debe ser un proceso planeado con antelación, puesto que tan importante es la intervención terapéutica como la preventiva. Desde esta perspectiva, el estudio de Jonasson et al. ${ }^{5}$ expone que las enfermeras son capaces de dar una sensación de seguridad y conexión para adaptarse a la nueva vida en HD, aliviando parte de la fragilidad experimentada. Este es un claro ejemplo de cómo las enfermeras se preocupan por facilitar los procesos de transición que experimentan los pacientes, sin embargo, nuestro estudio se limitó porque el tiempo de estancia de los participantes en HD fue en torno a 2-3 meses, tal vez si la vivencia se hubiera prolongado en el tiempo, se hubiera desarrollado una relación más estrecha entre enfermera-paciente que facilitase el desarrollo de estrategias de afrontamiento para adaptarse a la nueva terapia.

La vida en HD fue, en parte, comparable a la descripción que Schumacher y Meleis ${ }^{25}$ realizan de la última etapa de la transición: reformular la identidad. Esta fase simbolizó una vida con nuevos roles y responsabilidades, y que en la presente investigación se manifestó por sentirse preso, sentirse dependiente respecto a otros, o en pérdidas y limitaciones que tuvieron que aceptar (Hurst et al. ${ }^{29}$ y Clarkson et al. ${ }^{2}$ ). Este desafío a la auto identidad, emergió como un hallazgo que implicó la pérdida de uno mismo como resultado de la incertidumbre y confusión que seguía a un evento de crisis: el traspaso de DP a HD, y movió a los participantes a luchar por encontrar un sentido de sí mismos, intentando recuperar el control, a pesar de las interrupciones en las relaciones cercanas y la vida diaria, para finalmente identificar la reconstrucción de la identidad mediante cambios en el estado de salud y en el patrón de comportamiento. Este aspecto coincidió con investigaciones que describen el proceso de transición, no sólo como un movimiento o paso entre dos puntos, sino más bien como un momento de reorientación y/o transformación interna que la persona atraviesa para incorporar el nuevo cambio en su vida, mediante integración o adaptación a nuevos roles, habilidades o conductas ${ }^{15,30,31}$.

Las experiencias de cambios en el cuerpo y en la mente cuando el paciente inicia diálisis por primera vez se evidencian en los estudios de Mesa - Melgarejo et al. ${ }^{1}$ y Clarkson et al. ${ }^{2}$, y estos fueron identificados en nuestro estudio, pero partiendo del contexto que los participantes transitaron de una modalidad dialítica 
a otra, lo que evidenció que inicialmente ya habían experimentado este tipo de cambios en su vida, y en el momento de la transición, lo cambios venideros se tradujeron en una vivencia de mayor transcendencia, no obstante, no se han encontrado estudios cualitativos que corroboren esta afirmación pero existen investigaciones enfocadas a situaciones similares de transición analizadas por Meleis et al. ${ }^{32}$, como las que discuten la transición de la salud a la enfermedad, como el diagnóstico de un cáncer, a otras que se centran en las transiciones de la vida como convertirse en padres, o el retiro del mundo laboral, y las cuales exponen la vivencia de la transición como una experiencia donde las personas tienden a ser más vulnerables ya que están expuestos a más riesgos que pueden afectar a su salud.

Los participantes no se reconocieron en situaciones sociales habituales, su carácter se tornó más irascible y ello minó el contacto social con otros, incorporándose una identidad de enfermo, con la que no se identificaban en DP. Calvey et al. ${ }^{33}$ lo describen como la definición de un nuevo sentido de sí mismo, y Guerra-Guerrero et al. ${ }^{34}$ lo enfocan hacia una lucha diaria para ajustarse a la nueva realidad. Por otro lado, la red familiar surgió como el principal sistema de apoyo en la transición, lo que permitió ir sobrellevando la HD en el día a día, con el fin de optimizar el estado de salud y en consecuencia la calidad de vida, en consonancia a lo que afirma Bayhakki et al. ${ }^{4}$, un individuo no debe separarse y desconectar del mundo social que le rodea, ya sea familia o amigos.

Por todo ello podemos decir que la experiencia de transición de DP a HD emergió como una vivencia de gran vulnerabilidad que implicó profundos cambios en la vida de los participantes, supuso un traspase de roles, desde el autocuidado aprendido en DP a la dependencia familiar y sanitaria surgida en HD, no obstante, transitar de DP a HD significó redefinir la identidad para intentar alcanzar un pleno estado de bienestar, trasluciéndose la necesidad de determinar el papel del profesional en el manejo de las diferentes transiciones que acompañan a los pacientes en el transcurso de la enfermedad renal.

\section{Agradecimientos}

Estamos muy agradecidos a todos los pacientes que han participado en el estudio por compartir sus experiencias con el equipo de Diálisis Peritoneal del HUCA.
Recibido: 27 diciembre 2018

Revisado: 12 enero 2019

Modificado: 25 enero 2019

Aceptado: 18 febrero 2019

\section{Bibliografía}

1. Mesa-Melgarejo L, Carrillo-Algarra AJ, Castiblanco RA, Reina LM, Ávila TM. Terapias de sustitución de la función renal: Metaestudio y síntesis de evidencias cualitativas. Aquichan. 2017;17(3):328-52.

2. Clarkson KA, Robinson K. Life on dialysis: $A$ lived experience. Nephrol Nurs J. 2010; 37(1):29-35.

3. Tong $A$, Lesmana B, Johnson DW, Wong G, CampbeII $D$, Craig JC. The perspectives of adults living with peritoneal dialysis: Thematic synthesis of qualitative studies. Am J Kidney Dis. 2013;61(6):873-88.

4. Bayhakki, Hatthakit U. Lived experiences of patients on hemodialysis: A meta-synthesis. Nephrol Nurs J. 2012; 39(4):295-304.

5. Jonasson K, Gustafsson L-k. You live as much as you have time to: The experience of patients living with Hemodialysis. Nephrol Nurs J. 2017;44 (1):35-41.

6. Ley básica reguladora de la autonomía del paciente y de derechos y obligaciones en materia de información y documentación clínica. Ley 41/2002, de 14 de noviembre. Boletín Oficial del Estado, $n^{\circ}$ 274, (15/11/2002). [Consultado 5 Dic 2018]. Disponible en: https://www.boe.es/buscar/doc.php?i$d=B 0 E-A-2002-22188$

7. Botella J. Aspectos éticos, sociales y económicos del tratamiento de la insuficiencia renal. Nefrología. 1993;13(Supl 5):S38-40.

8. Arrieta J, Rodríguez-Carmona A, Remón C, Pérez-Fontán $\mathrm{M}$, Ortega $\mathrm{F}$, Sánchez-Tornero JA et al. La diálisis peritoneal es la mejor alternativa coste-efectiva para la sostenibilidad del tratamiento con diálisis. Nefrología. 2011;31(5):505-13. 
9. Liberek T, Renke M, Skonieczny B, Kotewick k, Kowalewska J, Chmielewski M et al. Therapy outcome in peritoneal dialysis patients transferred from haemodialysis. Nephrol Dial Transplant. 2009;24 (9):2889-94.

10. Covic A, Bammens B, Lobbedez T, Segall L, Heimbürger 0 , Van Biesen $W$ et al. Educating end-stage renal disease patients on dialysis modality selection: A clinical advice from the European Renal Best Practice (ERBP) Advisory Board. Nephrol Dial Transplant Plus. 2010;3(3):225-33.

11. Sauvé C, Vandyk AD, Bourbonnais FF. Exploring the facilitators and barriers to home dialysis: A scoping review. Nephrol Nurs J. 2016;43(4):295-308.

12. Sadala MLA, Bruzos GAS, Pereira ER, Bucuvic EM. Patients' experiences of peritoneal dialysis at home: A phenomenological approach. Rev Lat Am Enfermagem. 2012;20(1):68-75

13. Meleis A. Transitions theory. In: Smith $M$, Parker $M$, editors. Nursing theories and nursing practice. $4^{a}$ ed. Philadelphia: F. A. Davis Company; 2015. p. 361-80.

14. Meleis A, Trangenstein P. Facilitating Transitions: Redefinition of the nursing mission. Nurs Outlook.1994;42:255-9.

15. Fex A, Flensner G, Ek AC, Söderhamn 0. Health-illness transition among persons using advanced medical technology at home. J Caring Sci. 2011;25 (2):253-61.

16. Boissinot L, Landru I, Cardineau E, Zagdoun E, Ryckelycnk JP, Lobbedez T. Is transition between peritoneal dialysis and hemodialysis really a gradual process? Perit Dial Int. 2013;33(4):391-7.

17. Szeto CC, Kwan BC, Chow KM, Pang WF, Kwong VW, Leung $C B$, et al. Outcome of hemodialysis patients who had failed peritoneal dialysis. Nephron Clin Pract. 2010; 116(4):c300-6.

18. Covic A, Bammens B, Lobbedez T, Segall L, Heimbürger 0 , Van Biesen $W$ et al. Educating end-stage renal disease patients on dialysis modality selection: A clinical advice from the European Renal Best Practice (ERBP) Advisory Board. Nephrol Dial Transplant Plus. 2010; 3(3):225-33.
19. Mendes A, Bastos F, Paiva A. A pessoa com Insuficiência Cardíaca. Factores que facilitam/dificultam a transição saúde/doença. Rev Enf Ref. 2010;3(2): 7-16.

20. Martin-McDonald K, Biernoff D. Initiation into a dialysis-dependent life: an examination of rites of passage. Nephrol Nurs J. 2002;29(4):347-52.

21. Lundmark M, Erlandsson LK, Lennerling A, Almgren $M$, Forsberg $A$. Health Transition after lung transplantation - a grounded theory study. J Clin Nurs. 2016; 25(15-16):2285-94.

22. Contreras de la Fuente HS, Castillo-Arcos LC. Fenomenología: una visión investigativa para enfermería. Cult Cuid. 2016;44:15-24.

23. Hutchinson TA. Transitions in the lives of patients with end stage renal disease: a cause of suffering and an opportunity for healing. Palliat Med. 2005; 19:590-4.

24. Kaezer F, Valente G. Vivendo entre o pesadelo e 0 despertar: o primeiro momento no enfrentamento da diálise peritoneal. Esc Anna Nery. 2011; 15(1):39-46.

25. Schumacher K, Meleis A. Transitions: A central concept in nursing. J Nurs Sch. 1994;26(2):119-27.

26. Lindsay $H$, MacGregor C, Fry M. The experience of living with chronic illness for the haemodialysis patient: An interpretative phenomenological analysis. Health Soc Rev. 2014; 23(3):232-41.

27. Moran A, Scott A, Darbyshire P. Waiting for a kidney transplant: patients'experiences of haemodialysis therapy. J Adv Nurs. 2010;67(3):501-9.

28. Arman M, Rehnsfeldt $A$. The hidden suffering among breast cancer patients: A qualitative metasynthesis. Qual Health Res. 2003; 13 (4):510-27.

29. Hurst $H$, Summers $A$, Beaver $K$, Caress AL. Living with encapsulating peritoneal sclerosis (EPS): The patient's perspective. Perit Dial Int. 2014;34 (7):758-65.

30. Kralik D, Visentin K, Van Loon A. Transition: A literature review. J Adv Nurs. 2006;55(3):320-9. 
31. Meleis AI. Transitions theory: Middle range and situation specific theories in nursing Research and practice. New York: Springer Publishing Company; 2010.

32. Meleis AI, Sawyer LM, Im E0, Hilfinger Messias DK, Schumacher K. Experiencing transitions: An emerging middle-range theory. ANS Adv Nurs Sci. 2000;23(1):12-28.

33. Calvey D, Mee L. The Lived experience of the person dependent on haemodialysis. J Ren Care. 2011; 37(4):201-7.

34. Guerra-Guerrero V, Plazas MP, Camargo BL, Salas AV, González CG. Understanding the life experience of people in haemodialysis: Adherence to treatment and quality of life. Nephrol Nurs J. 2014;41 (3):289-97.

35. Bayhakki, Hatthakit U. Lived experiences of patients on hemodialysis: A meta-synthesis. Nephrol Nurs J. 2012;39(4):295-304.

Este artículo se distribuye bajo una Licencia Creative Commons Atribución-NoComercial 4.0 Internacional. https://creativecommons.org/licenses/by-nc/4.0/

\section{Open Access (c) (1) ( $)$}

